\title{
Los roles de la Escuela
}

\author{
The roles of the School
}

\author{
Carlos Javier Martínez González \\ Universidad Surcolombiana, Colombia \\ cajamar@usco.edu.co
}

\section{Introducción}

En concordancia con el Ministerio de Educación Nacional (MEN), el país está frente a una oportunidad única de darle un nuevo significado a la Educación en todos sus niveles, hoy cuando las posibilidades de pensar y construir un territorio alejado del conflicto guerrilla-Estado es algo inminente. Por ello, simposios, congresos, foros y demás eventos que abran espacios para la discusión deben ser alentados y contar con los aportes de los maestros, pero también de los administradores de la educación y de los generadores de políticas educativas, así como de profesionales de otras disciplinas.

Es en estos espacios en donde se puede dinamizar la capacidad deacción, de pensamiento y de transformación de la educación.

En este caso concreto, la verdadera importancia del Foro Educativo Nacional radica en su incidencia política por cuanto pone en la agenda social, educativa, ideológica, cultural y de decisión un asunto espinoso cual es el de la calidad de la educación y los roles, a la vez obstáculos y retos, que tiene la escuela, tanto desde sus expresiones curriculares como desde sus dimensiones humanas, ya que todo proceso educativo se da entre seres humanos, en la acción comunicativa que se manifiesta en los intersticios de la ciencia, del arte y de la vida cotidiana, como procesos de construcción colectiva de conocimiento y de sentido común.

Muchas voces ponen en duda el verdadero impacto de estos ejercicios de discusión, porque son considerados asuntos del 'establecimiento' en los que no hay nada que aportar, ni mucho menos discutir; creo que gran parte de esta suerte de 'desconfianza' surge en esencia desde algunos paradigmas (esos patroncitos del pensamiento) que nos sumergen en los temores ontológicos y esenciales del ser humano y que nos impiden dejar de lado esa visión paranoica del conflicto ideológico y nos conducen a vivir, como diría Estanislao Zuleta, (en su célebre 'Elogio de la dificultad') "en un mar de mermelada" $y$ en el amor que sentimos por las cadenas por miedo a la libertad. Como seres humanos, por nuestra misma estructura mental y genética, es dable que vivamos enfrentados a tres grandes temores: al cambio, al rechazo y al fracaso; pero, como seres humanos, estos temores son -a su vez- los que nos empujan al asombro, a la duda, a la curiosidad y a la búsqueda permanente de algo mejor, de ese horizonte de horizontes, de esos inéditos viables.

En consecuencia, discusiones como las suscitadas en este tipo de eventos deben poner en relieve y de modo autónomo y claro sus dimensiones éticas y políticas. Como afirma Tedesco, citado por Gadotti (en 'Historia de las ideas pedagógicas'), "la calidad de la educación y su mayor o menor dinamismo y eficiencia no tienen relación directa con el carácter público o privado de los establecimientos de enseñanza, y sí con la capacidad de llevar adelante una gestión autónoma". Pero además de la capacidad autónoma de acción (que en términos de Hanna Arendt no es sólo la capacidad de actuar, sino también la de pensar), deben ser señalados sus alcances éticos -en el sentido profundo dado por Sócrates al afirmar que el conocimiento correcto conduce a acciones correctas- y políticos -en las precisiones ofrecidas por Aristóteles al afirmar que la política pone orden en el caos y de acuerdo las partes en desacuerdo. 
La principal conclusión de arranque, a partir de los análisis hechos desde el MEN, tiene que ver con "la necesidad de revisar los propósitos, las pedagogías y los ambientes educativos para implementar procesos de enseñanza-aprendizaje significativos y pertinentes a la finalidad de formar en: la dimensión socio-emocional, la construcción de autonomía e identidad personal, la auto-identificación de talentos $e$ intereses formativos para seleccionar una ocupación, profesión y contar con las condiciones para la continuidad educativa, de tal manera que puedan afrontar y participar de los cambios sociales, culturales y productivos de la sociedad basada, hoy día, en el conocimiento y la información. Igualmente, establecer las competencias, que deben desarrollar los estudiantes en su calidad de ciudadanos y trabajadores del siglo XXI, asociadas a la adquisición de habilidades para el aprendizaje permanente $y$ la selección adecuada de información en distintos momentos, actividades y contextos de la vida".

Por tanto, se debe precisar y perfilar una visión que recoja la dimensión humana, conceptual, metodológica y técnica del currículo y de los roles de la escuela y por inclusión, del docente; se trata de pasar de esa idea de seres y sociedades 'competitivos' a una de seres y sociedades competentes y que, además, se debe tener en claro que la gran diferencia entre 'ricos' y 'pobres' no está dada exclusivamente en los capitales financieros, comerciales y productivos, sino esencialmente en las oportunidades de acceso y permanencia en la educación, la ciencia, el conocimiento y al desarrollo del pensamiento creativo.

Los eventos que discuten la filosofía de la educación, la eficacia delos modelos pedagógicos, la pertinencia de las didácticas, entre otros asuntos, deben convertirse en el espacio propicio para la construcción participativa de política pública hacia la modernización de la educación, en particular la Media y el tránsito a lo que hoy ha comenzado a ser llamada Educación Terciaria. Pero la incidencia política propuesta es dable mediante la reflexión sobre la identidad, sentido y función social de la educación y la escuela, sobre todo en relación con el tránsito de los jóvenes a la educación terciaria, al mundo del trabajo y al ejercicio de la ciudadanía, triada no solucionada hasta ahora y cuyos intereses particulares van, en muchos casos, en vías distintas.

Las perspectivas y realidades del país nos sitúan ante un debate obligado y urgente que permita orientar el currículo, tener mediana claridad en cuanto a las competencias que se han de formar en los estudiantes, revisar y ajustar las pedagogías para lograr aprendizajes significativos durante la educación media; analizar el rol de la docencia dedicada a la formación de los adolescentes en la educación media; identificar estrategias encaminadas a facilitar el tránsito de los egresados de la educación media a la educación terciaria $\mathrm{y}$ concertar con distintos actores y agentes educativos, locales, regionales y nacionales las orientaciones generales que han de integrar la política de Modernización de la Educación Media y el tránsito a la Educación Terciaria.

Es una magna tarea la que en estos días nos reúne, y debemos enfrentarla, como docentes, con 'Optimismo Crítico', es decir con la certeza que podemos encontrar un camino viable, pero sin renunciar al ejercicio siempre productivo y pleno de conflictos y contradicciones que nos procura la crítica y con la conciencia plena de que no todo depende de la labor a veces apostólica y doctrinaria del docente, sino que se deben dar otras condiciones para no caer en la trampa de cambiar las cosas para que nada cambie.

En consecuencia, nos atrevemos a señalar por los menos ocho roles fundamentales y generales, que son obstáculos y retos de la escuela de hoy, sobre los que nos detendremos un poco, pero que no son más que una provocación, un intento por abrir troneras en nuestras creencias y prácticas educativas, útiles para la construcción del país nuevo que queremos.

\section{Los roles, obstáculos y retos de la escuela}

Uno: Aportar a la construcción de una Región Inteligente. Todos nuestros esfuerzos deben tener como horizonte la creación de una región inteligente, esto es una región que posea una masa crítica o una cantidad de personas 
cada vez más creciente y capaz de definir sus problemas y plantear alternativas viables de solución, en una dinámica propia que le permite ser autosostenible, autocrítica y propositiva. Esta idea de región inteligente encuentra su dinamismo en los procesos educativos y de aquí la importancia que adquiere dentro del contexto educativo hacer que el centro de la acción educativa esté en grupos de personas que deben gestar su potencial mediante visiones compartidas de desarrollo social, la superación de condiciones de marginamiento, la integración social y la reducción de factores de vulnerabilidad. La dificultad de este reto podría estar, entre otras cosas, en lo que De Certau (en 'Lo cotidiano y la cultura'), ha señalado en cuanto a que los códigos que utilizamos en la gramática de la producción no siempre son los mismos códigos que utilizamos en la gramática de la interpretación. En sentido metafórico, construir región inteligente implica construir gramáticas compartidas por todos y acercarnos al concepto de 'ética solidaria', que no es más que reconocer cómo nuestros actos afectan a los demás.

Dos: Ofrecer más y mejor cobertura y equidad. Desde el 2002, cuando los gobiernos se han comprometido a mejorar de manera notable la cobertura y equidad en la educación, en cuanto a cantidad y calidad, se ha ido avanzando en todos los niveles, pero en particular en el nivel de educación Secundaria, cuyos crecimientos en número de matriculados han sido de más del 13\% en educación básica secundaria y de más del $10 \%$ en educación media. A pesar de este logro innegable, un número importante de personas en edad escolar sigue sin llegar al final de la educación media, en particular en las zonas rurales. Además, el asunto de la calidad se ha visto frenado por las grandes inversiones que se deben realizar, no sólo en infraestructura física $\mathrm{y}$ en recursos educativos, sino en la formación avanzada del recurso humano, pues recordemos que los niveles de educación continua de nuestros docentes están muy por debajo de los registrados en países como Chile, México o Argentina.

Ya en el 2010, la Organización para la Cooperación y el Desarrollo Económicos (OCDE) alertó sobre esta situación y precisó (en serio análisis de la Educación Superior en Colombia, así existan dudas de tinte más ideológico que fáctico al respecto) que de los casi ocho millones y medio de jóvenes entre 15 y 24 años que están en el sistema educativo, "el $15.2 \%$ no completó la educación secundaria y no siguió estudiando; un $27.1 \%$ estaba todavía en la educación secundaria; un $23.8 \%$ salió de la educación secundaria, pero nunca entró en la educación superior; el 17.9\% ingresó en la educación superior y aún se encuentra en ella; el $14 \%$ entró en la educación superior, pero abandonó antes de la graduación; y el 2\% entró en la educación superior y se tituló". Así, nuestra gran tragedia nacional siguen siendo las barreras excluyentes, de todo tipo, en acceso y permanencia en el sistema escolar.

Se espera que la construcción de un nuevo país parta de un fuerte apoyo estatal para superar tales condiciones de atraso, marginalidad y pobreza, como también es esperable una actitud mucho más consistente de la masa docente nacional en cuanto a su contribución a la superación de las condiciones descritas.

Tres: Contribuir al mejoramiento de los estándares académicos básicos. Si bien es cierto que las pruebas diseñadas a nivel nacional o internacional que pretenden identificar el desarrollo de competencias generales y específicas de los estudiantes que terminan su ciclo de secundaria o universitario tienen mucho cuestionamientos, también lo es que los resultados de tales pruebas definen, no sólo el ingreso de muchos estudiantes al sistema de educación superior, sino el mismo escalafón de la institución educativa y afectan los índices de competitividad de las regiones, por lo cual el asunto no debe verse de manera reduccionista o demasiado simplificado.

Desde 2006, Colombia participa en el Programa para la Evaluación Internacional de Alumnos -PISA-, y a la fecha lo ha hecho en cuatro ocasiones, con resultados más o menos permanentes, es decir, con muy pequeños avances. PISA es una encuesta del conocimiento y las habilidades que se realiza cada tres años a estudiantes de 15 años y se ha diseñado para hacer comparaciones válidas entre países $\mathrm{y}$ culturas; tiende a centrarse en tres áreas básicas: 
lectura, matemáticas y ciencias; en cada edición, se expresa un énfasis y así es como PISA 2009 lo tuvo en lectura, la versión de 2012, en matemáticas y la de 2015 en ciencias.

La gran conclusión es que la calidad continúa siendo la principal urgencia de nuestra escuela; aunque sería injusto negar los avances del país, se debe señalar que los resultados están muy lejos de los de otros países e incluso tienden a estar en el grupo inferior de países de América Latina. Se destacan los avances en lectura y ciencias, pero se insiste en el atraso en el área de matemáticas, cuyos avances cuantitativos comparados entre 2006 y 2012 fue de apenas 1,1, muy distantes del 1,8 obtenido en ciencias y del 3,0, de lectura.

Cabe también destacar dos hechos claves que deben ser motivo de empuje decidido en las IES; uno, consistente en la relación directa que existe entre los mejores resultados obtenidos por aquellos estudiantes que hicieron por lo menos un nivel de Preescolar, en comparación con aquellos que ingresaron a la Primaria de una vez. Esta observación indica que en el campo que se discute, la formación y las posibilidades de acceso a la educación preescolar son definitivas en la construcción del país que queremos y que las universidades deben fomentar la existencia de programas académicos dirigidos a la formación de docentes en este nivel, con altos estándares de calidad. El otro, que las regiones de Manizales, Medellín, Bogotá y Cali están por encima del resto de las otras regiones colombianas, aunque con resultados muy distantes de los evidenciados en ciudades como Shanghái, Singapur, Hong Kong o Liechtenstein, para citar algunas de las ubicadas en el tope, lo que indica que los gobiernos locales son decisivos en el mejoramiento de la calidad de la educación, no solamente en cuanto a resultados de pruebas como estas, sino en lo relacionado con infraestructura, organización, recursos educativos y formación de docentes.

Por ello, se deben superar profundas brechas estructurales entre la ciudad y el campo, entre la educación de los niños y las niñas y en la consolidación de cobertura y calidad de la primera infancia.
En términos numéricos, los resultados promedios de la prueba PISA dice que en matemáticas fue de 494 puntos, en general, y en Colombia, de 376; en ciencias, el puntaje promedio general fue de 501, y en Colombia de 399, y en Lectura, el general fue de 496, y el del país, de 403. La escala de desempeño de PISA está construida de forma que para cada una de las tres áreas, la puntuación media se sitúa en torno a 500, con aproximadamente dos tercios de estudiantes que puntúan entre 400 y 600 puntos. Una diferencia de 39 puntos equivale a un año de escolaridad, por lo que un estudiante colombiano de 15 años, está 3 años escolares por debajo del promedio, en matemáticas; 2,6 años, en ciencias y 2,4 años, el lectura.

De este modo, es claro que en lectura, matemáticas y ciencias naturales, el sistema educativo colombiano está por debajo de los patrones esperados $\mathrm{y}$, en consecuencia, el reto de la acción docente es grande y prometedora en estos campos. Tal reto no se deriva de una prueba que puede tener serias críticas en su construcción y aplicación; en realidad, se construye porque las áreas mencionadas están en íntima conexión con el desarrollo consistente de la acción comunicativa, el pensamiento lógico, la capacidad de asombro y la necesaria indagación constante sobre el mundo y sus fenómenos sociales y naturales.

Cuatro: Desarrollar nuevas concepciones de currículo. El currículo tiene como fin plasmar una determinada concepción educativa en términos de lo individual, lo social y lo cultural. Existen muchas concepciones de educación pero lo importante es que haya congruencia entre el enfoque de educación y de currículo, que debe ser considerado en un proceso triple que implica tanto el desarrollo humano, como la interacción social y la creación y re-creación de cultura. El currículo es la concreción específica de una teoría pedagógica que pretende alcanzar niveles de acción efectiva y asegurar el aprendizaje y desarrollo de un grupo particular de alumnos para la cultura, época y comunidad de la que hacen parte.

Currículo es una tarea práctica que desarrolla una teoría pedagógica en el aula; es un 
sistema de organización de las actividades educativas y de aprendizaje en función de contenidos, métodos y técnicas didácticas que surgen de fuentes claras y palpables $y$, en consecuencia, se fundamenta en dimensiones políticas, psicológicas y epistemológicas. En la dimensión política, el currículo provee de elementos esenciales que se incorporan para garantizar que el proceso educativo responda a las necesidades, demandas y expectativas sociales; en la dimensión psicológica busca y aporta información relativa a las expectativas que se tienen en cuanto al tipo de ser humano que se desea formar, en términos de valores, habilidades, destrezas y del perfil ciudadano que se propicia mediante el proceso educativo, $\mathrm{y}$, por último pero no menos importante, en la dimensión epistemológica integra las áreas del saber en función del análisis de las condiciones que hacen posible el aprendizaje.

Por tanto, se debe pensar en un currículo holístico que supere la sinonimia excluyente de plan de estudios y se agoté allí. No podremos seguir construyendo currículos fragmentados, llenos de hojarasca, 'contenidistas', muchas veces irrelevantes y pocas veces pertinentes, en vez de estar enraizados en argumentos, plantear objetivos claros, coherentes y viables, postular temas articulados, estimar tiempos, aclarar dimensiones y competencias, precisar recursos necesarios y determinar criterios e instrumentos de evaluación.

Cinco: Adecuar del currículo a lo esencial y significativo. Lo expuesto con anterioridad, debe conducir a que, aunque parezca un ejercicio mecánico, es hora de revisar la carga de contenidos temáticos que debe cursar (y ¿aprender?) un alumno promedio en un colegio promedio de un curso promedio: ve 16 asignaturas al año, que constan de 4 unidades temáticas, lo que nos dice que se enfrenta a 64 unidades temáticas, que a su vez se dividen en 4 subtemas, lo que arroja un total de 256 temas sobre los que el alumno debe dar cuenta y razón; en otras palabras, si dividimos 256 temas en 9 meses -el año escolar o lectivo- tendríamos 28 temas por mes, o sea jun poco más de un tema nuevo cada día!. A esto, agreguemos las tareas de todo tipo, que en más de las ocasiones deseadas son el reflejo de lo que saben los padres o de la capacidad económica de la familia para pagar un profesor particular que 'refuerce' algo que, en esencia, debería ser motivo de gestación de dinámicas muy interesantes al interior del aula. Así, urge revisar la carga 'contenidista' de las estructuras curriculares, adecuarla a lo esencial, analizar necesidades y expectativas del entorno y reforzar la utilidad social del conocimiento.

Seis: Evaluar para y desde aprendizaje, no desde la enseñanza. Quizá debido a ese afán por desarrollar múltiples contenidos sin un hilo conductos, como docentes nos hemos centrado en la enseñanza y nos sentimos conformes con enseñar, pero en contadas ocasiones ponemos el foco en el aprendizaje; en consecuencia, y en sentido metafórico, enseñamos a hacer torta de chocolate, pero en la evaluación exigimos que nos hagan un ponqué de matrimonio. Esto nos lleva a discutir algo primordial como es el valor pedagógico y evaluativo de las tareas escolares que, tradicionalmente, han sido vistas como una labor que se debe realizar dentro de un tiempo determinado y con el uso de unos recursos específicos, para cumplir una exigencia o demanda académica. No obstante, queremos ir un pocomás allá deesta acepción rutinaria y llevar el concepto y dinámica de las tareas escolares tanto al sujeto, como al vínculo que se forja en su realización y al grupo. En otras palabras, la tarea escolar debe ser trabajo en conjunto que vincula a varios alumnos en torno a propósitos comunes; posibilidad de establecer vínculos cognitivos, sociales y emocionales; acción grupal en constante proceso de retroalimentación; pretexto perfecto para la unión de la teoría y la práctica; proceso de aprendizaje creativo que genera conocimientos cada vez más profundos; oportunidad de aprender a pensar, de aprender a hacer y de aprender a aprender; espacio mental, físico y temporal que nos permita pasar de la profunda competitividad al desarrollo y fortalecimiento de competencias esenciales en la formación de seres humanos éticos, socialmente útiles y constructores de ciudadanía.

Siete: Darles nuevos significados a los roles del docente. La docencia se entiende como un 
entramado, un tejido que está articulado en y desde el arte, la ciencia, la tecnología y la vida cotidiana en función del conocimiento y en aplicación tanto teórica como práctica. Como es obvio suponer, su desempeño se evidencia en un campo bastante complejo como es el educativo, ámbito en donde con más ahínco se sienten las tensiones de un mundo que atraviesa por una profunda crisis estructural cuyas consecuencias aún no son previstas del todo.

En una investigación adelantada en las Universidades del Tolima, la Amazonia y la Surcolombiana, con el patrocinio de ASCOFADE, en la que tuvimos la fortuna de participar, se les preguntó a docentes y estudiantes cuáles deberían ser los atributos del docente y sus resultados fueron concordantes con lo obtenido por investigadores como Abell, Acevedo y Grossman (citados en el programa Todos a Aprender: para la transformación de la calidad educativa, del MEN) en por lo menos cinco aspectos, así:

a) Orientación y motivación del aprendizaje, que incluye el uso de metodologías adecuadas para alcanzar los objetivos de aprendizaje, la calidad de su comunicación verbal y no verbal así como la capacidad de suscitar interés por la asignatura; fuera de esto, la aplicación de prácticas pedagógicas que promuevan el espíritu crítico y la expresión de ideas y opiniones relacionadas con la asignatura.

b) Relaciones interpersonales, cimentadas en una comunicación docente-alumno dentro de un ambiente de cordialidad y respeto, en la flexibilidad para aceptar la diversidad de opinión y sentimientos de los alumnos y respeto real por sus diferencias de género, raza y situación socioeconómica junto con la receptividad a las críticas y sugerencias de los estudiantes y una firme contribución a la formación de valores individuales y colectivos.

c) Gestión del conocimiento, cuyos atributos se expresan en el orden y consistencia teórica y metodológica en el desarrollo de las clases, el cumplimiento de horarios de clase, de asesorías y de entrega de calificaciones, al mismo tiempo que la capacidad para planificar adecuadamente el proceso educativo y establecer los objetivos claros, concisos y viables de aprendizaje que los estudiantes deben lograr.

d) Conocimiento disciplinar, que involucra atributos como el espíritu crítico frente a los avances científicos relacionados con la asignatura, el dominio de los contenidos que un docente desarrolla, el conocimiento de los materiales, documentos, artículos y demás información relacionada con su asignatura y la utilización del léxico o vocabulario apropiado de acuerdo con la naturaleza del curso.

e) Evaluación del aprendizaje, donde se pueden destacan 2 condiciones: por un lado, la adecuación entre el contenido de las pruebas de evaluación y los objetivos de la asignatura y, por otro, la claridad y consistencia en los criterios de evaluación.

En síntesis, estas líneas no son más que una provocación para que enfrentemos los retos de la escuela como sistema en la construcción del país que debe ser, desde una postura crítica pero optimista pues en caso contrario no deberíamos estar aquí y ahora asumiendo el compromiso y la responsabilidad de transformar mediante el ejercicio del de docentes. 